\title{
Erratum to: Distribution and phylogenetic diversity of $c b b M$ genes encoding RubisCO form II in a deep-sea hydrothermal field revealed by newly designed PCR primers
}

Shingo Kato $\cdot$ Michiyuki Nakawake $\cdot$

Moriya Ohkuma $\cdot$ Akihiko Yamagishi

Published online: 16 January 2014

(C) Springer Japan 2014

Erratum to: Extremophiles (2012) 16:277-283

DOI 10.1007/s00792-011-0428-6

In the original publication of this paper, in the sections "Primer design for $c b b M$ genes" and "PCR clone library analysis", the reverse primer name is incorrectly given as cbbM1226R which should be cbbM1126R.

Also in the section "Primer design for $c b b M$ genes", the expected amplicon size is incorrectly given as $800-900 \mathrm{bp}$ which should be 700-800 bp.

The online version of the original article can be found under doi:10.1007/s00792-011-0428-6.

S. Kato - M. Ohkuma

Japan Collection of Microorganisms, RIKEN BioResource

Center, 2-1 Hirosawa, Wako, Saitama 351-0198, Japan

S. Kato $\cdot$ M. Nakawake $\cdot$ A. Yamagishi $(\bowtie)$

Department of Molecular Biology,

Tokyo University of Pharmacy and Life Science,

1432-1 Horinouchi, Hachioji, Tokyo 192-0392, Japan

e-mail: yamagish@toyaku.ac.jp 